\title{
An STS Marker, Y202, Located on the Silene latifolia Y Chromosome between the Chromosomal Distal-end Satellite DNA and S/Y1
}

\author{
Naoko Fujita*, Kotaro Ishii and Shigeyuki Kawano \\ Department of Integrated Biosciences, Graduate School of Frontier Sciences, University of \\ Tokyo, Bldg. FSB-601, 5-1-5 Kashiwanoha, Kashiwa, Chiba 277-8562, Japan
}

Received March 11, 2008; accepted May 21, 2008

\begin{abstract}
Summary The pseudoautosomal region (PAR) is the chromosomal region where XY sex-chromosome pairing occurs. The boundary of the PAR delineates the XY pairing and non-pairing regions, but detailed information about this region is lacking. In this study, we analyzed the sequences adjacent to $S I X 1$ and SlY1 in the dioecious plant Silene latifolia. SlXI and SlY1, which are linked to the X and Y sex chromosomes, respectively, are thought to be closely linked to the PAR. The Sl-distalsatDNA is a satellite DNA found at the ends of $S$. latifolia chromosomes. The chromosomal distance between $S l X 1 / S l Y 1$ and Sl-distal-satDNA has not been examined. It was probably almost entire the PAR. However, the region between SlY1 and the Sl-distal-satDNA could be amplified in PCR. The nucleotide sequence between SIY1 and the Sl-distal-satDNA was determined following PCR amplification and compared to the sequence adjacent to $S I X 1$ using BAC clones. In addition, a new malespecific STS marker, Y202, was designed based on the sequence adjacent to SlY1. Y202 as well as SlY1 were mapped on the Y chromosome using a panel of deletion mutants and FISH analysis. Our data suggest that the sequences adjacent to $S I X I$ and SlYI do not simply follow a gradient of sequence divergence, a feature that was thought to allow the functioning of the PAR boundary. Therefore, recombination suppression in the XY chromosomes probably occurs via other mechanisms.
\end{abstract}

Key words PAR, STS marker, XY sex chromosomes, Silene latifolia, Sl-distal-satDNA.

$\mathrm{XY}$ sex chromosomes can be divided into recombination and non-recombination regions (Lahn et al. 2001). The chromosomal region where XY chromosome pairing occurs is called the pseudoautosomal region (PAR). The defining features of the boundary between the PAR and the recombination suppression regions are unknown. One possible mechanism of recombination suppression is sequence divergence. Since XY chromosome pairing occurs in the PAR (Scotti and Delph 2006), the PAR probably exhibits nearly $100 \%$ homologous sequence between the $\mathrm{X}$ and $\mathrm{Y}$ chromosomes, and sequences with lower homology are probably sufficient to cause recombination arrest.

Silene latifolia contains satellite DNA regions called Sldistal-satDNA (Silene latifolia chromosomal distal -end satellite DNA). These sequences are located on both ends of the X chromosome and one end of the Y chromosome (Buzek et al. 1997, Matsunaga et al. 1999, Garrido-Ramos et al. 1999, Kazama et al. 2003). The end of the Yq arm is known as the PAR, where the Sl-distal-satDNA is found (Lengerova et al. 2003, Kazama et al. 2003, 2006). Since FISH analysis using Sl-distal-satDNA as a probe has confirmed that it is located at the ends of chromosomes, the Sl-distalsatDNA is probably part of the PAR. Because $S I X 1$ (on the X chromosome) and $S l Y 1$ (on the Y chromosome) are the most homologous genes between the X and $\mathrm{Y}$ chromosomes (97\% similarity; Delichere et al. 1999), it has been proposed that they are closely linked to the PAR (Filatov et al. 2000, Zluvova et al. 2005, Filatov et al. 2005). We speculated that the similarity in sequence in the region between the $S l$-distal-satDNA and $S I X 1 / S l Y 1$ might increase gradually toward $100 \%$ near the

*Corresponding author, e-mail: 87302@ib.k.u-tokyo.ac.jp 
PAR, such that sequence diversity would cause recombination arrest. In addition, if the $S$ l-distalsatDNA is located in the PAR, and $S I X I$ and $S I Y 1$ are located proximal to the PAR, the nucleotide sequence between the $S l$-distal-satDNA and $S I X 1 / S l Y 1$ should include the boundary of the PAR.

We determined the sequence of the region between the Sl-distal-satDNA and SlY1 and compared it with the sequence adjacent to $S I X 1$. In addition to the nucleotide sequence adjacent to SlY1, we report a new male-specific STS marker.

\section{Materials and methods}

\section{Plant material}

An inbred Silene latifolia line, K, was produced by 11 generations of brother-sister mating to obtain genetically homogeneous populations. Plants were grown from seeds in pots in a regulated chamber at $23^{\circ} \mathrm{C}$.

\section{Primers and probes}

The following primers were used for PCR: SIX1/SIY1_P1 (5'-GGCCAGGCTCATTTTCAAGTAAATG-3'), SIY1_P2 (5'-GGCATACACAAACAAGCTAC-3'), X43R (5'-GACCTTCCGAACGGTTGAAA-3'; Sykorova et al. 2003), Y202F (5'-CAGGTTGTGTCACTGTGTGAAACTGA-3'), and Y202R (5'-CGGAAGACATTAACCCATTTGATAC-3'). For the FISH analysis probe, the primers SIX1/SIY1_P1 and FISH_Y202_R1 (5'-GCAAGCATCTGGGAGGATTA-3') were used.

\section{PCR and cloning}

PCR was performed on genomic DNA using Blend Taq (Toyobo). The PCR cycles were as follows: 3 min at $94^{\circ} \mathrm{C} ; 33$ cycles of $1 \mathrm{~min}$ at $94^{\circ} \mathrm{C}, 1 \mathrm{~min}$ at $63^{\circ} \mathrm{C}$, and $1 \mathrm{~min}$ at $72^{\circ} \mathrm{C}$; and $7 \mathrm{~min}$ at $72^{\circ} \mathrm{C}$.

\section{Southern blot analysis}

Genomic DNA was extracted from leaves using the Nucleon PhytoPure Kit (GE Healthcare). Genomic DNA was digested overnight at $37^{\circ} \mathrm{C}$ with HindIII (Takara Bio Inc.), and $15 \mu \mathrm{g}$ of the digested DNA were electrophoresed on 1\% agarose gels and blotted onto Biodyne B membrane (Pall). The membrane was hybridized with PCR-amplified probe and detected using the Alkphos Direct Nucleic Acid Labeling and Detection systems (GE Healthcare) according to the manufacturer's instructions.

\section{Chromosome preparations}

Root tips were harvested, pretreated in ice-cold water $\left(0^{\circ} \mathrm{C}\right)$ for $24 \mathrm{~h}$, and then fixed in acetic acid-ethanol (1:3) for $24 \mathrm{~h}$. After washing in distilled water for $10 \mathrm{~min}$, meristematic portions of the root tips were treated at $37^{\circ} \mathrm{C}$ for $80 \mathrm{~min}$ in $1.5 \%$ Cellulase $\mathrm{YC}$ (Kikkoman) and $1.0 \%$ Pectolyase Y-23 (Kikkoman) in $2 \times \mathrm{SSC}(\mathrm{pH} 4.0)$ containing $10 \mathrm{nmol} / \mathrm{l}$ EDTA. The root portions were then washed carefully with distilled water. Two or three portions were transferred to a glass slide, macerated in a drop of acetic acid: ethanol (1:3) using fine forceps, and air-dried.

\section{Fluorescence in situ hybridization (FISH)}

A DNA probe for the fragment containing SlY1, Y202, and the adjacent sequence was amplified and labeled using the PCR DIG Labeling Kit (Roche). A probe for the Sl-distal-satDNA (KpnI) was labeled with biotin-16-dUTP (Roche) as described by Kazama et al. (2003). Chromosomal DNA was denatured at $75^{\circ} \mathrm{C}$ for $50 \mathrm{~s}$ in $20 \times \mathrm{SSC}$ containing $70 \%$ formamide. The chromosome preparations were then dehydrated immediately in $70 \%$ ethanol at $-20^{\circ} \mathrm{C}$ for $5 \mathrm{~min}$ and $100 \%$ ethanol at room temperature for $5 \mathrm{~min}$. The preparations were then treated with acetone for $30 \mathrm{~min}$ 
at room temperature. Each slide was loaded with $9 \mu \mathrm{l}$ of a hybridization mixture containing $13.3 \mathrm{ng} / \mu \mathrm{l}$ of Y202 probe DNA, $6.6 \mathrm{ng} / \mu 1$ of the $S l$-distal-satDNA probe DNA, $50 \%$ formamide, $10 \%$ dextran sulfate, and $2 \times \mathrm{SSC}$. The slides were washed at $37^{\circ} \mathrm{C}$ in $2 \times \mathrm{SSC}$, and then counterstained with DAPI II (Vysis, Chicago, IL).

Biotin-labeled probe (Sl-distal-satDNA) was detected using Anti-Alexa Fluor 488 (Molecular Probes). For the digoxigenin-labeled probe (Y202), detection was performed using sheep anti-DIGrhodamine (Roche) followed by donkey anti-sheep Cy3 (Jackson ImmunoResearch Laboratories) for signal amplification. Fluorescence signals were observed using a Leica Q550 cytogenetic workstation (Leica Microsystems, Wetzlar, Germany) equipped with a black-and-white CCD camera (CoolSNAP HQ, Nippon Roper, Tokyo, Japan) along with the SpectraVysion filters Spectrum Green, Spectrum Gold, Spectrum Red, Spectrum Fred, and Spectrum DAPI, respectively (Vysis). The detected images were colored and processed using Photoshop version 5.02 J (Adobe, San Jose, CA).

\section{Results}

\section{The Sl-distal-satDNA is located adjacent to SlY1}

The primer P1 hybridizes to both $S I Y 1$ and $S I X 1$, since it was designed based on a region that is $100 \%$ homologous to both $S l Y 1$ and $S l X 1$. In PCR using the P1 primer and a primer specific for X43.1 (X43R), a fragment was amplified only from the male template. SlX1 should exist in both males and females, as both have the $\mathrm{X}$ chromosome. Several different primers, designed according to the $S I X 1$ nucleotide sequence, were used in place of P1, but these primers amplified no fragment from the female template. Therefore, there is probably Sl-distal-satDNA adjacent to SlY1 but no Sldistal-satDNA adjacent to $S I X 1$. Direct sequencing confirmed that the fragment amplified in this PCR includes the Sl-distal-satDNA on one end and SlY1 on the other end. Inverse PCR was used to extend the nucleotide sequence adjacent to the $S l$-distal-satDNA. Approximately $2 \mathrm{~kb}$ were sequenced in the region adjacent to $S I Y 1$.

To compare this sequence with the sequence adjacent to $S I X 1$, a bacterial artificial clone (BAC) containing $S l X 1$ was isolated, and approximately $2 \mathrm{~kb}$ adjacent to $S l X 1$ were sequenced. A schematic diagram of the sequences is shown in Fig. 1. It is clear that a Sl-distal-satDNA is proximal to SlY1, but not to SlX1. Between the X and Y chromosomes, there are several "strata" that differ in sequence divergence. In the first stratum, the region about $200 \mathrm{bp}$ from $S l Y 1 / S l X 1$, the sequence divergence is $81.7 \%$, and about one unit of $S l$-distal-satDNA is followed by the first stratum in the $\mathrm{Y}$ chromosome. The nucleotide sequence in the first stratum contains telomere-like sequences, including the inverted telomere sequence 5'-GGGATTT-3' (the telomere sequence is 5' TTTAGGG-3'). Multiple units of the inverted telomere sequence are present near degenerate telomere sequences. A male-specific primer set, Y202, was designed to hybridize within the first stratum.

The second stratum is a region with $92.9 \%$ sequence identity, as shown in Fig. 1. In this stratum, the nucleotide sequences in the $\mathrm{X}$ and $\mathrm{Y}$ chromosomes are highly homologous. The third stratum has low sequence identity (approximately 25\%). The third stratum on the Y chromosome contains a nucleotide sequence homologous to an Arabidopsis thaliana $\beta$-galactosidase gene, but in the $\mathrm{X}$ chromosome, this region contains a sequence homologous to a gene encoding an $A$. thaliana retrotransposon-like protein.

\section{Y202 and SlY1}

To confirm that Y202 is located next to SlY1, genomic Southern hybridization was performed (Fig. 2). $S l X 1$ probe DNA was amplified from the BAC 26d11c. Since $S l X 1$ is highly homologous to $S l Y 1$, the $S I X 1$ probe hybridizes to both $S I Y 1$ and $S I X 1$. A fragment of about $7.5 \mathrm{~kb}$ showed hybridization in both male and female, but the fragment length was slightly different. Because non-radioisotopic Southern hybridization was performed, the SlX1 signal was too faint to observe in male 


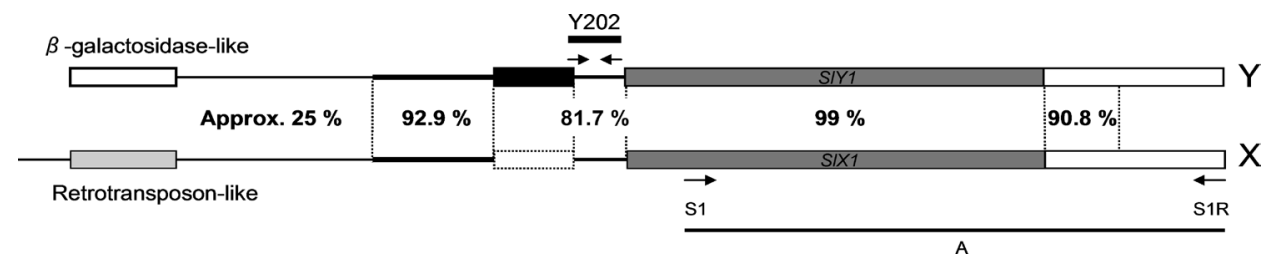

Fig. 1. Schematic diagram of sequences adjacent to $S l Y I$ and $S I X I$ on the $\mathrm{X}$ and $\mathrm{Y}$ chromosomes. The percentages between the chromosomes show the sequence similarity in each region. Note that there are relatively similar sequences next to $S l Y 1$ and $S I X 1$, but no $S l$-distal-satDNA (black box) is found in the region adjacent to $S I X 1$. The sequence adjacent to the $S l$-distal-satDNA (on the left side) can be divided into two regions of highly similar (92.9\%) and non-similar (less than $25 \%$ ) sequences. The locations to which the primers used for PCR hybridize are indicated by arrowheads. The location of the new male-specific marker Y202 is indicated by a bold black line. The fragment A used in the Southern blot analysis in Fig. 2 is shown.

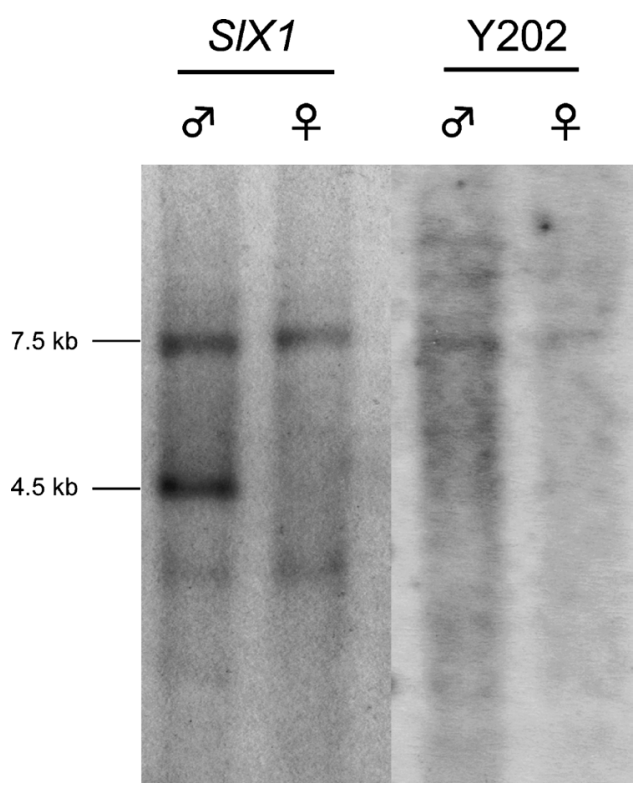

Fig. 2. Southern blot analysis with either $S l X 1$ or Y202 as a probe. Genomic DNA from male (ð) or female ( $($ ) plants was digested with HindIII and analyzed by Southern blotting. The $S I X 1$ probe was amplified from a BAC containing $S I X 1$. The $S I X 1$ probe hybridizes to both SIXI and SlY1, since $S l X 1$ is highly similar to $S l Y 1$. Note that the signal at $7.5 \mathrm{~kb}$ in male corresponds to $S l Y 1$, whereas the signal in female corresponds to $S l X 1$. The $S I X I$ signal at $7.5 \mathrm{~kb}$ in male is too faint to be observed, because a non-radioisotopic method was used. The same DNA samples were hybridized with the Y202 probe. Signals corresponding to SIXI and Y202 were detected on the same fragment $(7.5 \mathrm{~kb})$ in male, indicating the proximity of Y202 to SlY1. The Y202 signal was weak because the sequence adjacent to $S l X 1$ is $81.7 \%$ similar (Fig. 1) to the region of Y202 in the $\mathrm{Y}$ chromosome. (because females have XX chromosomes and males have XY chromosomes, the SIXI signal is doubly intense in females), and there were two bands of about $7.5 \mathrm{~kb}$ in male. Radioisotopic Southern hybridization performed by Delichere et al. (1999) showed two SlY1 signals at fragments of 12 and $4.5 \mathrm{~kb}$, and a $12-\mathrm{kb}$ SlX1 signal was also detected. The differing fragment sizes of the $S l X 1$ and SlYI signals may explain the two signals at $7.5 \mathrm{~kb}$ in the Southern hybridization shown in Fig. 2. A Y202 signal of $7.5 \mathrm{~kb}$ was detected in male. A weaker Y202 signal is seen in female at $7.5 \mathrm{~kb}$, because there is a region of $81.7 \%$ homology located adjacent to $S I X 1$. It is expected that the Y202 probe hybridized to this region. In fact, the Y202 probe hybridized to a slightly larger fragment in female than in male. The length of the fragment that hybridized to Y202 in female DNA is the same as that of the $S I X 1$ signal detected in female. These data confirm the proximity of Y202 to SlY1.

\section{Mapping of Y202 using Y-chromosome dele- tion mutants}

To map Y202 on the Y chromosome, PCR was performed on a panel of $S$. latifolia Y-chromosome deletion mutants (Fig. 3). Y202 is deleted in two hermaphroditic mutants, and four mutants with a deletion of SlY1 have been reported. Y202 should be located proximal to SlY1. However, our results suggested the presence of SlY1 in two mutants (UH8 and MS63) in which Y202 was also scored as present. This result was confirmed by PCR using the 


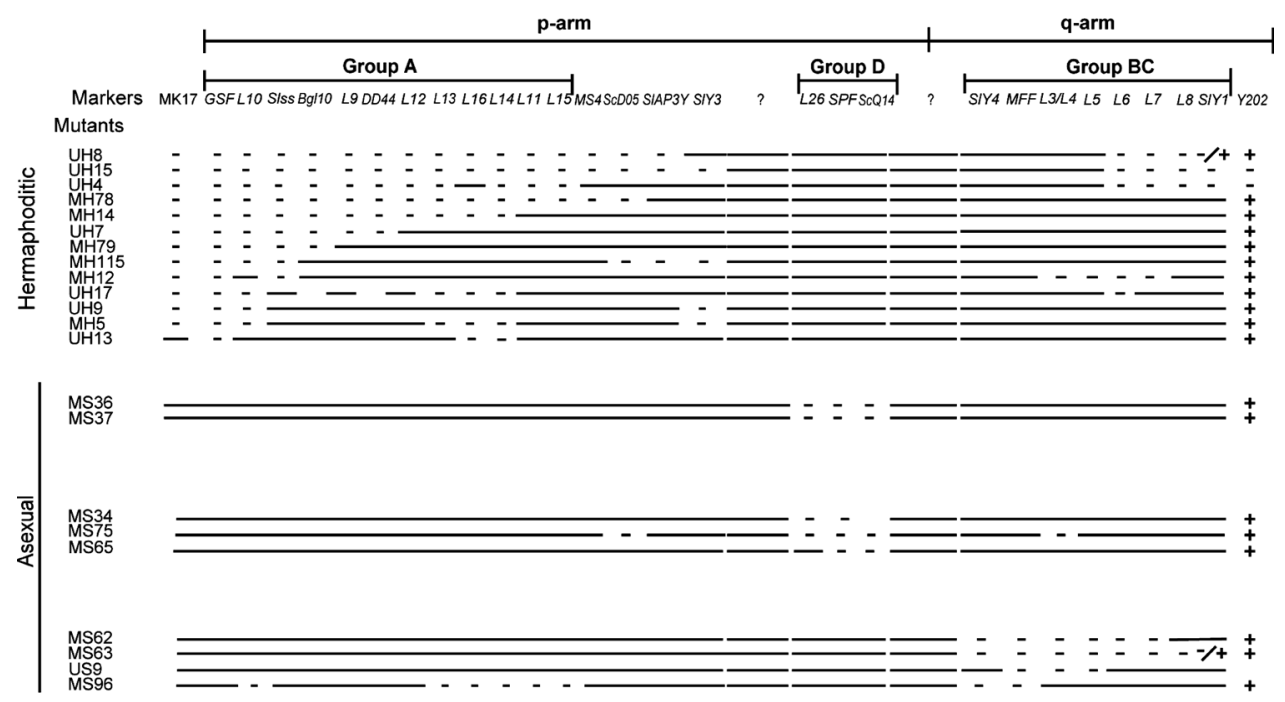

Fig. 3. Mapping of $\mathrm{Y} 202$ on the $\mathrm{Y}$ chromosome. The relative position of $\mathrm{Y} 202$ was determined by PCR with genomic DNA from Y-chromosome deletion mutants. The map is arranged according to Hobza et al. (2006), with mutant identifiers on the left vertical axis and marker loci on the top horizontal axis. Y-deletion mutants were scored for the presence $(+)$ or absence $(-)$ of the Y202 PCR product. The scores for the UH8 and MS63 mutants are indicated as $-/+$, because the PCR results suggest the presence of Y202 (+) (data not shown) but the results of a previous experiment indicated its absence (-). US9 genomic DNA was not analyzed, due to its being out of stock.
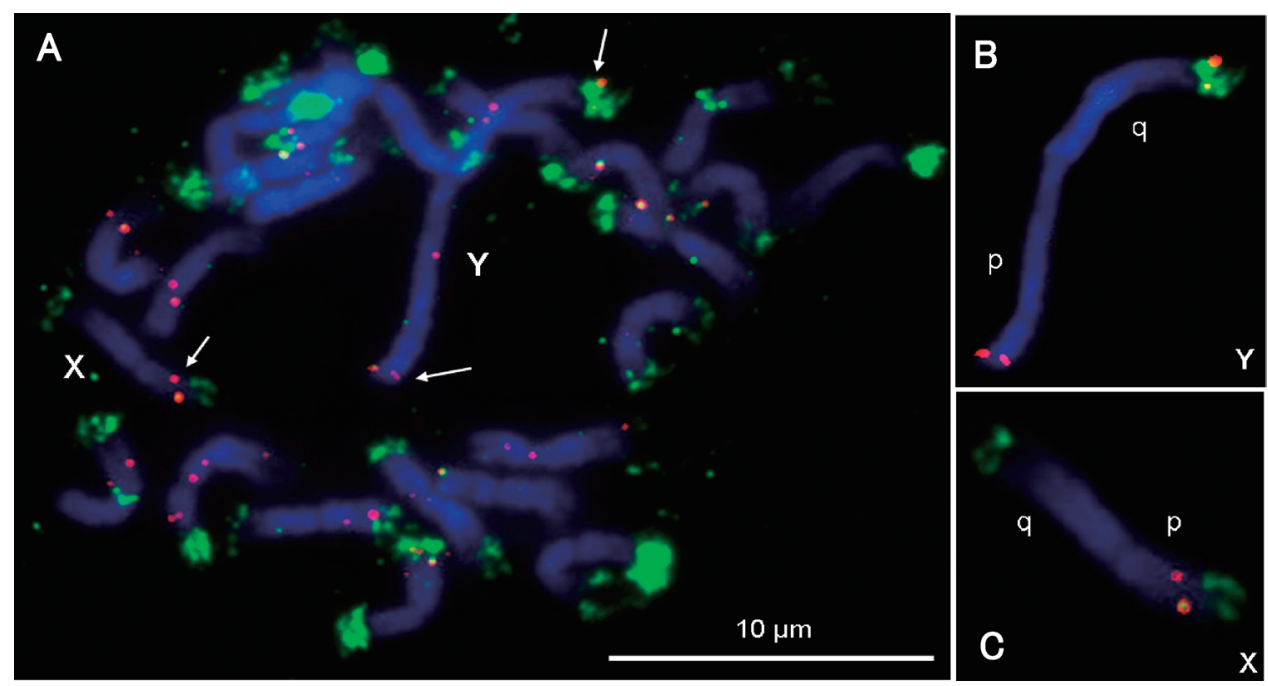

Fig. 4. FISH analysis of Y202. (A) Male metaphase chromosomes were hybridized with a DIG-labeled DNA probe, Y202, which covers almost the entire sequence on the Y chromosome shown in Fig. 1, and with a biotin-labeled KpnI subfamily probe (Kazama et al. 2003). The chromosomes were counterstained with DAPI. Red signals indicate the Y202 probe, and green signals indicate the KpnI probe. Arrows represent signals corresponding to Y202 and/or SlY1. (B) Trimmed and enlarged Y chromosome from A. The side containing the KpnI subfamily signal is the q arm, and the other end of the chromosome is the $\mathrm{p}$ arm. Because most of the Y202 probe DNA is SlY1, the signal on the end of the p arm probably corresponds to a SlY1 homolog. (C) Trimmed and enlarged X chromosome from A. The part of the chromosome containing the Y202 signal is labeled the $\mathrm{p}$ arm. 
primers S1Y1_P2 (specific for SlY1) and Y202R (specific for Y202) (data not shown), demonstrating the presence of the region covering SlY1 and Y202. Thus, the presence of SlY1 in UH8 and MS63 is unclear (shown as $+/-$ in Fig. 3).

\section{FISH analysis of SIY1 and Y202}

To visualize the chromosomal location of Y202, FISH analysis was carried out. The length of the Y202 fragment is $202 \mathrm{bp}$, which is too short for use in FISH detection. Therefore, the probe DNA that was used is approximately $8 \mathrm{~kb}$ in length and includes Y202 as well as SlY1, almost the entire region of the $\mathrm{Y}$ chromosome shown in Fig. 1. Two signals were detected on the Y chromosome, at the ends of the Yq and Yp arms, whereas one signal was detected on the $\mathrm{X}$ chromosome (Fig. 4). The Sl-distal-satDNA marker KpnI (Kazama et al. 2003) was used as the chromosomalend marker (green signals). Since the $K p n I$ signal appears on the upper end of the $\mathrm{Y}$ chromosome in Fig. 4, this end is the Yq arm, and the other side, which shows no KpnI signal, is the Yp arm.

\section{Discussion}

\section{The Sl-distal-satDNA is dispersed on the ends of chromosomes}

FISH analysis with the Sl-distal-satDNA as a probe resulted in strong signals on the chromosome ends. To estimate the length of the Sl-distal satDNA on the Y chromosome, we determined the percentage of the signal of the Sl-distal satDNA in relation to the entire Y chromosome using FISH. The average percentage of the Sl-distal satDNA signal was $7 \%$ that of the Y chromosome (data not shown). Because $7 \%$ of the total size (570 Mb; Liu et al. 2004) is $39.9 \mathrm{Mb}$, the estimated length of the $S l$-distal satDNA is about $40 \mathrm{Mb}$. It is also known that the $S l$-distal satDNA exists as an island (i.e., 1-2 units) (unpublished data). The Sl-distal satDNA may exist not only as a cluster but also as dispersed short units. It is likely that the $S l$-distal satDNA is located proximal to $S l X 1$ and SlYI because the estimated length of the Sl-distal satDNA could be the major part of the PAR on the Y chromosome, and it might be located near the PAR boundary. To test whether the Sl-distal satDNA is closely linked to $S I X 1 / S l Y 1$, PCR was carried out. Interestingly, the results revealed one $S l$-distal satDNA adjacent to $S l Y 1$, but no $S l$-distal satDNA adjacent to $S l X 1$. The sequences adjacent to SlY1 (labeled Y202 in Fig. 1) and SlX1 include inverted telomere and degenerate telomere sequences. Telomeres are repetitive sequences found at the tips of chromosomes. The $S l$-distal satDNA is also found on the ends of chromosomes in S. latifolia. These regions probably arose due to chromosomal rearrangements. The Sl-distal satDNA may have been inserted only on the Y chromosome, or deleted only in the $\mathrm{X}$ chromosome following chromosomal rearrangement.

\section{A new male-specific STS marker, Y202}

To create a new male-specific STS marker, we first focused on the nonhomologous region between the $\mathrm{X}$ and $\mathrm{Y}$ chromosomes (to the left in Fig. 1). We designed several sets of primers and tested their male specificity, but no male-specific primer set was found. This is probably because there is a homologous region in the X chromosome that is not located near SlX1. This region may have been created following chromosomal inversion.

We therefore designed a male-specific STS marker in the next least homologous region and named it Y202 (because it amplifies a fragment of 202 bp from the Y chromosome). Nucleotide sequencing and Southern hybridization confirmed that Y202 is located next to SlY1. Therefore, if SlY1 is closely linked to the PAR, which is on the Yq arm, Y202 is located on the Yq arm. However, as Delichere et al. (1999) have mentioned, there is at least one SlY1 homolog in the Y chromosome. These results were also seen in the Southern hybridization (Fig. 2): probing using a $S l X 1$ probe resulted in a male-specific signal of $4.5 \mathrm{~kb}$ (Fig. 2). This fragment is probably the $S l Y 1$ homolog. Because the nucleotide sequence of the SlY1 homolog has not been determined, it is un- 
known whether Y202 is located next to the SlY1 or SlY1 homolog.

FISH analysis allows the visualization of chromosomal locations. Our FISH analysis, using a probe containing the Y202 and SlY1 sequences, resulted in two signals (red; Fig. 4). Since SlY1 comprises most of the probe DNA (approximately $6 \mathrm{~kb}$ of $S l Y 1$ sequence in the probe of $8 \mathrm{~kb}$ ), one of the two signals is thought to be the SlY1 homolog. This FISH experiment showed that the region containing SlY1 and Y202 is located at the end of Yp or Yq, and the SlY1 homolog is located on the other side.

\section{Sequence divergence and $X Y$ chromosome pairing}

Sequence diversity is a possible cause of suppression of XY chromosome pairing. If this were the case, the similarity between the $\mathrm{X}$ and $\mathrm{Y}$ chromosomes would be important in determining the PAR boundary. Our sequencing results, however, are inconsistent with this theory. Bergero et al. (2008) suggested that SlY1 is located on the Yp arm, according to an analysis of a panel of deletion mutants. SlY1 may be located on the end of the Yp arm instead of the Yq arm. However, if so, both Y202 and the Sldistal satDNA must be located on the Yp arm. There has been no report of the existence of the Sl-distal satDNA on the Yp arm. We therefore suggest that Y202 is located on the end of the Yq arm.

\section{Acknowledgements}

We are grateful to T. Arai for technical assistance. This work was supported by a Grant-in-Aid for Exploratory Research to S.K. (number 18657001) from the Ministry of Education, Culture, Sports, Science and Technology (MEXT).

\section{References}

Bergero, R., Charlesworth, D., Filatov, D. A. and Moore, R. C. 2008. Defining regions and rearrangements of the Silene latifolia Y chromosome. Genetics 178: 2045-2053.

Buzek, J., Koutnikova, H., Houben, A., Riha, K., Jonousek, B., Siroky. J., Gran, S. and Vyskot, B. 1997. Isolation and characterization of X chromosome-derived DNA sequences from a dioecious plant Melandrium album. Chromosome Res. 5: 57-65.

Delichere, C., Veuskens, J., Hernould, M., Barbacar, N., Mouras, A., Negrutiu, I. and Moneger, F. 1999. SlY1, the first active gene cloned from a plant Y chromosome, encodes a WD-repeat protein. EMBO J. 18: 4169-4179.

Filatov, D. A. 2000. Lox variability in a Y-linked plant gene and its implications for Y-chromosome evolution. Nature 404: 388-390.

— 2005. Substitution rates in a new Silene latifolia sex-linked gene, Slss X/Y. Mol. Biol. Evol. 22: 402-408.

Garrido-Ramos, M. A., de, la, Herran, R. and Ruiz, Rejon, C. 1999. A subtelomeric satellite DNA family isolated from the genome of the dioecious plant Silene latifolia. Genome 42: 422-446

Hobza, R., Hrusakova, P., Safar, J., Bartos, J., Janousek, B., Zluvova, J., Michu, E., Dolezel, J. and Vyskot, B. 2006. MK17, a specificmarker closely linked to the gynoecium suppression region on the Y chromosome in Silene latifolia. Theor. Appl. Genet. 113: 280-287.

Kazama, Y., Sugiyama, R., Matsunaga, S., Shibata, F., Uchida, W., Hizume, M. and Kawano, S. 2003. Organization of KpnI family of chromosomal distal-end satellite DNAs in Silene latifolia. J. Plant Res. 116: 317-326.

-, - , Suto, Y., Uchida, W. and Kawano, S. 2006. The clustering of four subfamilies of satellite DNA at individual chromosome ends in Silene latifolia. Genome 49: 520-530.

Lahn, B. T., Pearson, N. M. and Jegalian, K. 2001. The human Y chromosome, in the light of evolution. Nat. Rev. Genet. 2: 207-216.

Lengerova, M., Moore, R. C., Grant, S. R., and Vyskot, B. 2003. The sex chromosomes of Silence latiofolia revisited and revised. Genetics 165: 935-938.

Matsunaga, S., Kawano, S., Michimoto, T., Higashiyama, T., Nakao, S., Sakai, A. and Kuroiwa, T. 1999. Semi-automatic laser beam microdissection of the Y chromosome and analysis of the Y chromosome DNA in a dioecious plant, Silene latifolia. Plant Cell Physiol. 40: 60-68.

Sýkorová, E., Cartagena, J., Horáková, M., Fukui, K. and Fajkus, J. 2003. Characterization of terlomere-subtelomere junctions in Silence latifolia. Mol. Genet. Genomics 269: 13-20.

Zluvova, J., Janousek, B., Negrutiu, I. and Vyskot, B. 2005. Comparison of the X and Y chromosome organization in Silence latifolia. Genetics 170: 1431-1434. 